\title{
Contenidos y habilidades incluidas en cuestiones de evaluación sobre nutrición humana
}

\section{Content and skills included in evaluation on human nutrition issues}

\author{
Juan Carlos Rivadulla López, Susana García Barros, Cristina Martínez Losada \\ Departamento de Pedagoxía e Didáctica. Universidade da Coruña
}

\begin{abstract}
Resumen
En este trabajo se analizan las cuestiones de evaluación propuestas por maestros en formación de la Universidade da Coruña para el $3^{\circ}$ ciclo de Educación Primaria sobre el concepto/finalidad de nutrición humana. Se pretende conocer los contenidos que evalúan y las habilidades que exigen las cuestiones propuestas. La recogida de datos se realizó mediante una actividad formativa incluida en el tema de evaluación. Los resultados muestran que los participantes tienden a evaluar aspectos referidos a la función de nutrición asociada a la intervención de órganos/sistemas y suelen demandar habilidades como la identificación de características y la descripción de las mismas.
\end{abstract}

Palabras clave: educación primaria, formación inicial, evaluación, habilidades, nutrición humana.

\begin{abstract}
This paper discusses the evaluation questions proposed by teachers in training of the Universidade da Coruña to the 3 rd cycle of primary education on the concept/purpose of human nutrition. It is to know the contents that evaluated and the skills demanded by the questions proposed. Data were collected through a formative activity included in the subject of evaluation. The results show that participants tend to evaluate aspects relating to the function of nutrition associated with the intervention of organs/systems and often demanding skills such as the identification of characteristics and description of the same
\end{abstract}

Keywords: primary education, initial training, evaluation, skills, human nutrition.

\section{Introducción}

La evaluación es entendida por el profesorado como una "valoración" (Casanova, 2002), considerando que les sirve para detectar los puntos fuertes y débiles de los conocimientos del alumnado (Wang, Kao, y Lin, 2009), para informar al alumnado y a sus familias sobre esos puntos y para recuperar, así, los aprendizajes no adquiridos (Blanco Loro, 2010). En la investigación realizada por Castillo (2004) se pueden destacar tres aspectos clave: qué, cuándo y cómo piensan los/as profesores/as de Educación Primaria que evalúan. Así, sobre los aspectos que son tenidos en cuenta por el profesor a la hora de evaluar a sus alumnos suelen estar el esfuerzo y el trabajo personal del alumno, aunque la mayoría cree y confía en la objetividad de los instrumentos tradicionales, es decir, el examen escrito (Bartholomew, Osborne, y Ratcliffe, 2004; González Rodríguez, 2005). Los/as docentes no están de acuerdo con hacer un único examen por trimestre, si no que considera importante evaluar "continuamente, en cada sesión de clase". Para ello, la observación del trabajo en clase es el procedimiento, técnica e instrumento que más valora el profesorado a la hora de evaluar a sus alumnos/as, conclusión a la que también llegó Blanco Loro (2010) en su investigación.

Por otra parte, los profesores tienen que tomar decisiones fundamentadas $\mathrm{y}$ reflexivas sobre los contenidos que deben impartirse en el aula, lo cual no siempre es fácil. En este trabajo nos centramos en los contenidos sobre la nutrición humana que han de seleccionarse para Educación Primaria.

Así, la "nutrición humana", como función vital, es un tópico central dentro del núcleo conceptual de la Biología, que se incluye como objeto de aprendizaje reiterado a lo largo de la educación obligatoria. De hecho, el currículo de Ciencias de todos los niveles educativos contempla el estudio de la nutrición humana, plateándose su estudio de forma progresiva, es decir, con un nivel de profundidad creciente (Sanmartí, 2002; Rivadulla López, García Barros, y Martínez Losada, 2012). Concretamente, el currículo de Educación Primaria de la Comunidad Autónoma de Galicia (Decreto, 2007) incluye como contenidos a enseñar el reconocimiento de la nutrición como función vital, la identificación de todos los aparatos y sistemas del cuerpo humano, los hábitos de higiene y de vida saludable y la aceptación y práctica de las normas sociales referidas a la salud, higiene y alimentación.

En este sentido, numerosos estudios indican que los/as alumnos/as suelen mostrar grandes dudas (Carvalho, Silva, Lima, y Coquet, 2004; Rowlands, 2004; Cakici, 2005; Núñez, Mazzitelli, y Vázquez, 2007; Banet y López, 2010; García Barros, Martínez Losada, y Garrido, 2011), ya que aunque desde pequeños/as, niños y niñas son conscientes de la necesidad de los alimentos para vivir, para crecer..., tienen muchas y variadas explicaciones sobre la digestión, la respiración,... (Banet, 2001), estos suelen mostrar muchas carencias educativas en cuanto a estos temas. Pero no sólo los alumnos muestran dudas al 
respecto, si no que trabajos recientes realizados con futuros docentes han detectado problemas para definir y diferenciar entre alimentación y nutrición (Ejeda Manzanera, 2009), así como para reconocer las funciones específicas de los diferentes tipos de nutrientes, siendo las funciones plástica y reguladora las más desconocidas (Rodrigo Vega, Ejeda Manzanera, y González Barberá, 2010; Daza Rosales, Arrieta Vergara, Ríos Carrascal, y Crespo Rojas, 2012).

En Educación Primaria es importante que el alumnado adquiera una visión integral y unificada de nutrición en general y de la humana en particular. Esta última constituye un proceso vital que tiene lugar en las células y que consiste básicamente en el intercambio de materia y energía que el individuo realiza con el medio, lo que conlleva a su transformación. Esta idea sintética, desde el punto de vista científico, puede servir de referente para la ciencia escolar (García Barros y Martínez Losada, 2005; Pujol, 2003), que además debe integrarse en el concepto general más amplio de ser vivo. De hecho, el modelo de ser vivo constituye un modelo fundamental en el campo de la Biología que debe ser enseñado en el aula (Cañal, 2003; De las Heras y Jiménez Pérez, 2011), existiendo secuencias didácticas específicas en este sentido (Cañal, 2005; Garrido y Martínez, 2009).

En concreto, a través de este trabajo pretendemos conocer la importancia que otorgan los maestros en formación a la evaluación de aspectos relacionados con el concepto/finalidad de la nutrición humana, y las habilidades que exigen las cuestiones de evaluación que elaboran.

\section{Método}

Teniendo en cuenta la finalidad del estudio se optó por un enfoque metodológico cualitativo (Yin, 2006; Sabariego, Massot y Dorio, 2012) mediante una actividad realizada por los maestros en formación en el marco de la asignatura de la "Didáctica de las Ciencias Experimentales". Esta actividad se realizó en el horario habitual (módulos de dos horas).

A los participantes se les indica que la actividad que van a realizar, además de servir cómo actividad formativa, va a formar parte de una investigación $\mathrm{y}$, para ello, se les pide su consentimiento, garantizándoles la confidencialidad de los datos aportados. Durante su realización se promueve un ambiente cordial y distendido. Las actividades se recogieron en papel, para posteriormente proceder a su análisis.

\section{Participantes}

En este estudio han participado 29 pequeños grupos de dos/tres maestros en formación que cursaban la Diplomatura de Maestro de Educación Primaria en la Universidade da Coruña, en el curso académico 20102011. Esta muestra resulta coherente con la investigación cualitativa, pues responde a la accesibilidad de los participantes en el momento de la realización de la misma (McMillanm y Shumacher,
2005), y su número es suficiente, ya que en este tipo de metodología no es factible ni necesaria una muestra amplia (Stake, 2007).

\section{Instrumentos o Materiales}

Como instrumento de recogida de datos se utilizaron las propias actividades que realizan los futuros maestros durante su etapa formativa en el marco de esa asignatura, después de abordar el tema de ¿qué y cómo evaluar en Educación Primaria? Se trataba de una actividad de aplicación en la que debían elaborar instrumentos específicos con objeto de evaluar el aprendizaje de unos hipotéticos alumnos de tercer ciclo de Educación Primaria $\left(5^{\circ}\right.$ y $\left.6^{\circ}\right)$ en relación a la nutrición humana. Cabe destacar que este alumnado recibió formación específica sobre la nutrición humana en la asignatura de carácter científico "Introducción a las Ciencias de la Vida y de la Tierra".

\section{Procedimiento}

Los futuros docentes plantearon cuestiones de evaluación referidas a distintos aspectos relacionados con la nutrición humana. Cada cuestión puede incluir preguntas referidas a más de un aspecto o habilidad. Para analizarlas, se organizaron en torno a cuatro dimensiones relativas a este tópico: a) el concepto $y$ finalidad de la nutrición, b) los cuatro sistemas que intervienen en la nutrición humana, c) la alimentación y la salud, $y \quad d) \quad l a$ interrelación entre la alimentación/nutrición humana y el medio.

En esta ocasión nos centraremos únicamente en el estudio de las cuestiones referidas a la dimensión sobre el concepto y finalidad de la nutrición, que fueron formuladas por un total de 26 grupos. Para ello, dentro de esta dimensión se establecieron categorías que responden a distintas ideas clave: NO: Referencias genéricas; N1: Función restringida a la alimentación; N2: Función asociada a la intervención de órganos/sistemas; N3: Función a nivel organismo y N4: Función a nivel celular. A su vez, respecto a cada pregunta se identificaron las habilidades cognitivas y cognitivo lingüísticas que incluyen. Dentro de estas últimas estarían aquellas que se ponen en marcha para analizar y comprender la información recibida y que se expresan oralmente o por escrito (Ds -Descripción-, Df -Definición-, Ex -Explicación- y $J$-Justificación-...), mientras que dentro de las cognitivas estarían las que no requieren expresarse ni oralmente ni por escrito (IC Identificación de características- y Er-Establecimiento de relaciones-).

En primer lugar se analizaron las aportaciones de forma global, tanto en lo que se refiere a las ideas clave, como a las habilidades que incluyen en las cuestiones planteadas. A continuación se realizó un análisis individualizado de dichas cuestiones, con objeto de identificar cada idea clave con las habilidades que se asocian a cada una de ellas. 


\section{Resultados}

El análisis global de las aportaciones que realizan los 26 grupos participantes en relación a la nutrición (ver Figura 1), muestra que la gran mayoría (21 grupos) plantea cuestiones relativas a la función de la nutrición asociada a la intervención de órganos/sistemas (idea clave N2). Además, 13 grupos incluyen alguna cuestión sobre la función de la nutrición a nivel organismo (idea clave N3) y 10 lo hacen sobre referencias genéricas en relación con la nutrición (idea clave N0). Solo un grupo de participantes plantea cuestiones referidas a la función de la nutrición restringida a la alimentación (N1) y a la función de la nutrición a nivel celular (N4).

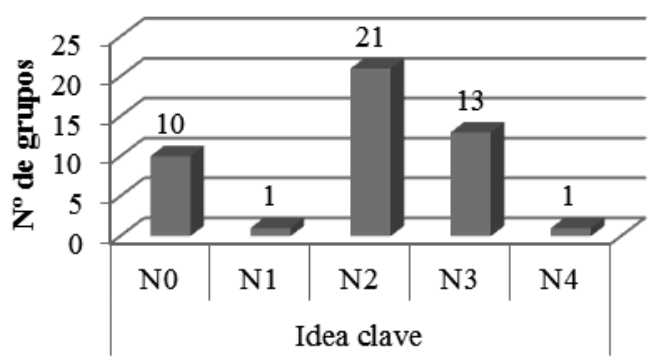

Figura 1. $\mathrm{N}^{\mathrm{o}}$ de futuros maestros que tienen en cuenta las distintas ideas clave en las cuestiones de evaluación

Por otra parte, la mayoría de los participantes solicitan en sus cuestiones el uso de habilidades cognitivolingüísticas (Figura 2), sobre todo la descripción (13 grupos), la explicación (11 grupos) y la definición (10 grupos) y en menor medida la justificación (2 grupos). Otros grupos también solicitan habilidades cognitivas, concretamente la identificación de características (13 grupos) y el establecimiento de relaciones (3 grupos).

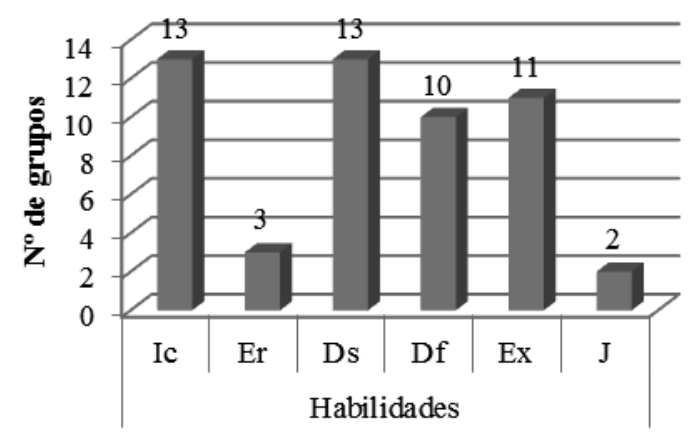

Figura 2. $\mathrm{N}^{\mathrm{o}}$ de futuros maestros que tienen en cuenta las distintas habilidades en las cuestiones de evaluación

Un análisis más detallado de las aportaciones que realiza cada grupo muestra que 12 de ellos atienden a una determinada idea clave, preferentemente la N2 (8 grupos) (Tabla 1). Otros 9 grupos consideran dos ideas clave, centrándose mayoritariamente en función de la nutrición asociada a la intervención de órganos/sistemas (N2). Los demás grupos (5) trabajan en sus cuestiones tres ideas clave. En cuanto al tipo de habilidades que exigen en las cuestiones, 10 grupos se centran en una, siendo la identificación de características (Ic) la más frecuente (4 grupos). Otros 9 grupos exigen dos tipos de habilidades (mayoritariamente la explicación y la justificación -3grupos-). Por su parte, 5 grupos exigen tres tipos de habilidades, mientras que solo 1 grupo exige cuatro o seis habilidades.

Tabla 1.

$N^{o}$ de ideas clave y de habilidades que cada grupo de maestros en formación incluye en las cuestiones de evaluación.

\begin{tabular}{|c|c|c|c|c|c|c|}
\hline$N^{o}$ & $\begin{array}{l}\text { Idea } \\
\text { Clave }\end{array}$ & \multicolumn{2}{|c|}{$\begin{array}{c}\text { Total } \\
(n=26)\end{array}$} & Habilidades & \multicolumn{2}{|c|}{$\begin{array}{l}\text { Total } \\
(n=26)\end{array}$} \\
\hline \multirow{4}{*}{$\begin{array}{c}1 \\
\text { tipo }\end{array}$} & $\mathrm{N} 2$ & 8 & \multirow{4}{*}{12} & Ic & 4 & \multirow{4}{*}{10} \\
\hline & N3 & 4 & & $\mathrm{Er}$ & 1 & \\
\hline & -- & -- & & Ds & 2 & \\
\hline & -- & -- & & Df & 3 & \\
\hline \multirow{6}{*}{$\begin{array}{c}2 \\
\text { tipos }\end{array}$} & $\mathrm{N} 0+\mathrm{N} 2$ & 5 & \multirow{6}{*}{9} & Ic+Ex & 1 & \multirow{6}{*}{9} \\
\hline & $\mathrm{N} 0+\mathrm{N} 3$ & 1 & & $\mathrm{Ic}+\mathrm{Df}$ & 1 & \\
\hline & $\mathrm{N} 2+\mathrm{N} 3$ & 2 & & $\mathrm{Ic}+\mathrm{Ds}$ & 1 & \\
\hline & $\mathrm{N} 2+\mathrm{N} 4$ & 1 & & Ds+Ex & 2 & \\
\hline & -- & -- & & Ds+Df & 1 & \\
\hline & -- & -- & & $\mathrm{Ex}+\mathrm{J}$ & 3 & \\
\hline \multirow{4}{*}{$\begin{array}{c}3 \\
\text { tipos }\end{array}$} & $\begin{array}{c}\mathrm{N} 0+\mathrm{N} 2 \\
+\mathrm{N} 3\end{array}$ & 4 & \multirow{4}{*}{5} & $\mathrm{Ic}+\mathrm{Ds}+\mathrm{Df}$ & 2 & \multirow{4}{*}{5} \\
\hline & $\begin{array}{c}\mathrm{N} 1+\mathrm{N} 2 \\
+\mathrm{N} 3\end{array}$ & 1 & & 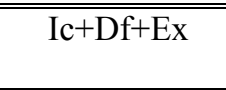 & 1 & \\
\hline & --- & -- & & Ic+Ds+Ex & 1 & \\
\hline & ב-- & -- & & Er+Ds+Df & $\overline{11}$ & \\
\hline $\begin{array}{c}4 \\
\text { tipos }\end{array}$ & -- & -- & & $\overline{\mathrm{Ic}+\mathrm{Ds}+\mathrm{Df}+\mathrm{Ex}}$ & & 1 \\
\hline $\begin{array}{c}6 \\
\text { tipos }\end{array}$ & -- & -- & & $\begin{array}{c}\mathrm{Ic}+\mathrm{Er}+\mathrm{Ds}+\mathrm{Df}+ \\
\mathrm{Ex}+\mathrm{J}\end{array}$ & & \\
\hline
\end{tabular}

Analizamos a continuación las cuestiones concretas aportadas por los distintos grupos. Tal como se muestra en la tabla 2, las habilidades cognitivas, y más concretamente la identificación de características y el establecimiento de relaciones, se solicitan sobre todo a través de la idea clave N2 (9 cuestiones en el caso de la primera habilidad-"A partir de la fotografía señala cuáles son los aparatos que intervienen en la nutrición”, 2 cuestiones en el caso de la segunda "Relaciona los órganos del cuerpo humano con los sistemas que intervienen en la nutrición humana”-).

Por su parte, en lo que se refiere a las habilidades cognitivo-lingüísticas, la descripción se exige mayoritariamente a través de la idea clave N2 (11 cuestiones - ¿Cuáles son los sistemas que intervienen en la nutrición? Identifica sus órganos y su función”-). Sin embargo, la definición se requiere sobre todo a través de la idea clave N0 (6 cuestiones - ¿Qqué entiendes por nutrición?”-). Así mismo, la habilidad cognitivo-lingüística de explicación, se demanda a través de la idea clave N3 (6 cuestiones - "Cuando llevamos a cabo el proceso de nutrición obtenemos energía de los alimentos. Podríais explicar ¿cómo 
obtenemos energía a partir de un bocadillo de jamón serrano?"'-). Por último, la habilidad de la justificación, que solo es demandada por dos participantes, lo hacen a través de la idea clave N0 (1 cuestión - ¿Es lo mismo alimentación que nutrición? Justifica tu respuesta”-) y N2 (1 cuestión "Buscad información acerca de los procesos que intervienen en la nutrición humana (digestión, respiración, circulación y excreción). ¿Creéis que alguno es más importante que otro? ¿Por qué? Justificadlo"-).

Tabla 2.

Relación entre las ideas clave y las habilidades solicitadas

\begin{tabular}{|c|c|c|c|c|c|c|}
\cline { 2 - 7 } \multicolumn{1}{c|}{} & \multicolumn{6}{c|}{ Tipos de habilidades } \\
\cline { 2 - 8 } \multicolumn{1}{c|}{} & Cognitivas & \multicolumn{4}{c|}{ Cognitivo-lingüisticas } \\
\hline Ideas clave & Ic & Er & $D s$ & $D f$ & $E x$ & $J$ \\
\hline N0 & & & 4 & 6 & 3 & 1 \\
\hline N1 & 1 & & & & & \\
\hline N2 & 9 & 2 & 11 & 3 & 3 & \\
\hline N3 & 2 & 1 & 2 & 2 & 6 & 1 \\
\hline N4 & 1 & & & & & \\
\hline
\end{tabular}

\section{Conclusión}

Aunque los futuros maestros formulan cuestiones variadas referidas a la evaluación de distintos aspectos relativos al concepto/finalidad de la nutrición humana, pocos son los que plantean cuestiones referidas a la función de la nutrición restringida a la alimentación y a la función de la nutrición a nivel celular. En términos generales, los estudiantes se centran en evaluar aspectos referidos a la función de la nutrición asociada a la intervención de órganos/sistemas.

Si bien, a través de la evaluación, los maestros en formación, en general, demandan habilidades cognitivas (de forma mayoritaria la identificación de características) y cognitivo-lingüísticas, estas últimas están especialmente centradas en la descripción, explicación y justificación, mientras que la habilidad de la justificación es muy poco considerada.

Además, los distintos grupos de estudiantes plantean mayoritariamente cuestiones referidas a una o dos ideas clave, demandando habitualmente una o dos habilidades.

Las conclusiones de este estudio indican que la enseñanza de la nutrición humana adolece de algunas deficiencias, como la insuficiente relación entre los contenidos que se enseñan, ya que la enseñanza de la nutrición se efectúa todavía de forma compartimentada y sesgada. Esto influye en que el alumnado no adquiera una visión integrada de la función de nutrición, la cual cosiste en un proceso vital que tiene lugar en las células y que se basa en el intercambio de materia y energía que el individuo realiza con el medio, lo que conlleva a su transformación (García Barros y Martínez Losada, 2005; Pujol, 2003). Por otra parte, la escasa solicitud de la justificación teórica resulta coherente con la priorización de habilidades poco exigentes, como la identificación de características y la descripción, mostrando así una visión restringida sobre el tipo de conocimientos que deben adquirir los niños de Educación Primaria. Ello puede deberse a la influencia de la formación científica previa que han recibido los estudiantes. No podemos olvidar que la enseñanza de este tema y concretamente los libros de texto, ampliamente utilizados como soporte de la misma, suelen promover esa visión restringida anteriormente señalada (Rivadulla López, 2013).

Estos datos constituyen una llamada de atención para la formación docente, pues muestran la insuficiencia de ésta para cambiar prioridades en lo que a la selección de contenidos a evaluar se refiere. Así, hay que indicar que el conocimiento científico ha de movilizarse para explicar procesos concretos e interesantes para el alumnado.

\section{Referencias}

Banet, E. (2001). Los procesos de nutrición humana. Madrid: Síntesis.

Banet, E., y López, C. (2010). ¿Cómo mejorar el desayuno de los escolares de Educación Primaria? Investigación en la Escuela, 71, 63-83. http://investigacionenlaescuela.es/articulos/71/R71 6. pdf

Bartholomew, H., Osborne, J., y Ratcliffe, M. (2004). Teaching students "ideas-about-science": five dimensions of effective practice. Science Education, 88(5), 655-682. https://cset.stanford.edu/sites/default/files/files/docum ents/publications/Bartholomew-

TeachingStudentsIdeasAboutScience.pdf

Blanco Loro, M. E. (2010). Políticas evaluativas del profesorado de Primaria, condicionantes de su actividad docente. Bordón, 62(1), 29-47. http://bddoc.csic.es:8080/detalles.html?id=750035\&b $\mathrm{d}=\mathrm{ISOC} \&$ tabla $=\mathrm{docu}$

Cakici, Y. (2005). Exploring Turkish upper primary level pupils' understanding of digestion. International Journal of Science Education, 27(1), 79-100. http://www.tandfonline.com/doi/abs/10.1080/0950069 032000052036\#.VZ0ptfntlBc

Cañal, P. (2003). ¿Qué investigar sobre los seres vivos? Investigación en la Escuela, 51, 27-38.

Cañal, P. (2005). La nutrición de las plantas: enseñanza y aprendizaje. Madrid: Síntesis.

Carvalho, G. S., Silva, R., Lima, N., y Coquet, E. (2004). Portuguese Primary School children's conceptions about digestion: identification of learning obstacles. International Journal of Science Education, 26(9),

1111-1130. http://www.journalsonline.tandf.co.uk/media/3pvxje0 m5kckvg893t33/contributions/1/n/t/g/1ntgjy119nfr3kj 3.pdf

Casanova, M. A. (2002). Manual de Evaluación Educativa. Madrid: La Muralla. 
Castillo, S. (2004). La práctica evaluadora del profesorado. Primaria y Secundaria. Granada: Grupo Editorial Universitario.

Daza Rosales, S. F., Arrieta Vergara, J. F., Ríos Carrascal, O., y Crespo Rojas, C. A. (2012). La digestión en la alimentación humana $\mathrm{y}$ sus implicaciones en la formación inicial de los profesores en didáctica de las ciencias naturales. Revista de la Red de Semilleros de Investigación de Santander, 1(1), 1-7.

De las Heras, M. A., y Jiménez Pérez, R. (2011). La enseñanza del ser vivo en primaria a través de una secuencia de estrategias indagatorias. Alambique: Didáctica de las Ciencias Experimentales, 67, 71-78. $\mathrm{http}$ ://auladesecundaria.grao.com/revistas/alambique/0 67-como-funciona-la-tierra/la-ensenanza-del-ser-vivoen-primaria-a-traves-de-una-secuencia-de-estrategiasindagatorias

Decreto $130 / 2007$, do 28 de xuño, polo que se establece o currículo da Educación Primaria para a Comunidade Autónoma de Galicia. Diario Oficial de Galicia, 132, 11666-11760.

Ejeda Manzanera, J. M. (2009). El conocimiento sobre alimentación en la formación inicial de maestros. Tesis doctoral. Departamento de Didáctica de las Ciencias Experimentales, Universidad Complutense, Madrid, España. http://eprints.ucm.es/8375/

García Barros, S., y Martínez Losada, C. (2005). La nutrición en textos escolares del último ciclo de Primaria y primero de Secundaria. Enseñanza de las Ciencias, Número extra. VII Congreso Internacional sobre Investigación en la Didáctica de las Ciencias.

García Barros, S., Martínez Losada, C., y Garrido, M. (2011). What do Children Aged Four to Seven Know about the Digestive System and the Respiratory System of the Human Being and of Other Animals? International Journal of Science Education, 33(15), 2095-2122.

http://www.tandfonline.com/doi/abs/10.1080/0950069 3.2010.541528\#.VZ0q8fntlBc

Garrido, M., y Martínez, C. (2009). ¿Qué enseñar sobre los seres vivos en los niveles educativos iniciales? Aula de Innovación Educativa, 183-184, 34-36. http://www.grao.com/revistas/aula/183_184competencia-artistica-y-cultural/que-ensenar-sobrelos-seres-vivos-en-los-niveles-educativos-iniciales

González Rodríguez, C. (2005). Análisis comparativo entre el currículo ofical y la programación de aula en enseñanza secundaria obligatoria, utilizando como marco conceptual la nutrición vegetal. Tesis doctoral. Departamento de Pedagoxía e Didáctica, Universidade da Coruña, A Coruña, España. http://ruc.udc.es/handle/2183/1069

Núñez, G., Mazzitelli, C., y Vázquez, S. (2007). ¿Qué saben nuestros alumnos sobre alimentación y nutrición? Revista Iberoamericana de Educación, 43(5), 1-8. http://www.rieoei.org/1758.htm

Pujol, R. M. (2003). Didáctica de las Ciencias en la Educación Primaria. Madrid: Síntesis.

Rivadulla López, J., García Barros, S., y Martínez Losada, C. (2012). ¿Qué dicen tratar los/as maestros/as del último ciclo de Educación Primaria sobre nutrición humana? Actas de los XXV Encuentros de Didáctica de las Ciencias Experimentales. Santiago de Compostela: Universidade de Santiago de Compostela.

Rivadulla López, J. C. (2013). El desarrollo del currículum desde la perspectiva del profesorado. La nutrición humana. Tesis Doctoral. Departamento de Pedagoxía e Didáctica, Universidade da Coruña, A Coruña, España. http://ruc.udc.es/bitstream/2183/11672/2/RivadullaLo pez_JuanCarlos_TD_2013.pdf

Rodrigo Vega, M., Ejeda Manzanera, J. M., y González Barberá, C. (2010). Una investigación en torno a las concepciones sobre alimentación en futuros maestros. Revista Complutense de Educación, 21(1), 189-207. http://revistas.ucm.es/index.php/RCED/article/view/R CED1010120189A

Rowlands, M. (2004). What do children think happens to the food they eat? Journal of Biological Education, 38(4), 167-171. http://eric.ed.gov/?id=EJ939789

Sanmartí, N. (2002). Didáctica de las Ciencias en la educación secundaria obligatoria. Madrid: Síntesis Educación.

Wang, J. R., Kao, H. L., y Lin, S. W. (2009). Preservice teachers' initial conceptions about assessment of science learning: the coherence with their views of learning science. Teaching and Teacher Education, 26(3), 522-529. http://www.sciencedirect.com/science/article/pii/S074 2051X09001395 